\title{
PROXIMAL-TYPE ALGORITHMS FOR SPLIT MINIMIZATION PROBLEM IN P-UNIFORMLY CONVEX METRIC SPACES
}

\author{
${ }^{1}$ C. IZUCHUKWU, ${ }^{2}$ G.C. UGWUNNADI, ${ }^{3}$ O.T. MEWOMO, ${ }^{4}$ A.R. KHAN, ${ }^{5}$ M. ABBAS.
}

\begin{abstract}
In this paper, we study strong convergence of some proximal-type algorithms to a solution of split minimization problem in complete $p$-uniformly convex metric spaces. We also analyse asymptotic behaviour of the sequence generated by Halpern-type proximal point algorithm and extend it to approximate a common solution of a finite family of minimization problems in the setting of complete $p$-uniformly convex metric spaces. Furthermore, numerical experiments of our algorithms in comparison with other algorithms are given to show the applicability of our results.
\end{abstract}

\section{INTRODUCTION}

Let $X$ be a geodesic space and $f$ be any real-valued function defined on $X$. If there exists a point $\bar{v} \in X$ such that $f(\bar{v})=\min _{v \in X} f(v)$, then $\bar{v}$ is called a minimizer of $f$ and is denoted by $\bar{v}:=\underset{v \in X}{\operatorname{argmin}} f(v)$ (that is, $\underset{v \in X}{\operatorname{argmin}} f(v)$ denotes the set of minimizers of $f$ ). The problem of finding such a point $\bar{v} \in X$ for which $f(\bar{v})=\min _{v \in X} f(v)$, is called a Minimization Problem (MP), which is very useful in optimization theory, convex and nonlinear analysis. Many authors have proposed some efficient and implementable algorithms and obtain some convergence theorems for solving MPs and some of their generalizations, (see for example, [21, 22, 23, 33, 35, 43, 49]). The Proximal Point Algorithm (PPA) is a well-known method for finding solutions of MPs. It was introduced by Martinet [32] and was further developed by Rockafellar [41] in Hilbert spaces. Rockafellar [41] proved that the PPA converges weakly to a minimizer of a proper convex and lower semicontinuous functional (to be defined in Section 2) and raised a very important question as to whether the PPA converges strongly or not. The question was resolved in the negative by Güler [21] who constructed a counterexample showing that the PPA does not necessarily converges strongly (see also [9, 10] for more counterexamples on this subject matter). In other words, except additional conditions are imposed on either the convex functional or on the underlying space, only weak convergence results for PPA are expected. In 2000, Kamimura and Takahashi [24] modified the PPA into Halpern-type PPA, so that its strong convergence is guaranteed. The study of PPA has been generalized from Hilbert spaces to differentiable manifolds, in particular, the Hadamard manifolds (Riemannain manifolds of nonpositive sectional curvature) see for example [20,30, 40] and the references therein. Bačák [6] continued along this line and introduced the PPA in Hadamard spaces (complete CAT(0) spaces) as follows: For arbitrary point $x_{1}$ in a Hadamard space $X$, define the sequence $\left\{x_{n}\right\}$ iteratively by

$$
x_{n+1}=J_{\mu_{n}}^{f}\left(x_{n}\right),
$$

where $\mu_{n}>0$ for all $n \geq 1$, and $J_{\mu}^{f}: X \rightarrow X$ is the Moreau-Yosida resolvent of a proper convex and lower semicontinuous functional defined by

$$
J_{\mu}^{f}(x)=\arg \min _{v \in X}\left(f(v)+\frac{1}{2 \mu} d^{2}(v, x)\right) .
$$

Bačák [6] proved that the PPA $\Delta$-converges (to be defined in Section 2) to a minimizer of $f$ under the assumption that $\sum_{n=1}^{\infty} \mu_{n}=\infty$ and that $f$ has a minimizer in $X$. Since then, different modifications of the PPA have been studied in Hadamard spaces, as well as in Hilbert and Banach spaces (see [4, 5, 44, 48] and the references therein).

The PPA have now been generalized to $p$-uniformly convex metric spaces, introduced by Noar and Silberman [34] in 2011 as follows: Let $1<p<\infty$, a metric space $(X, d)$ is called $p$-uniformly convex with parameter $c>0$

Key words and phrases. Split minimization problem, proximal point algorithm, $p$-uniformly convex spaces; resolvent; convex functions.

2010 Mathematics Subject Classification. 47H09; 47H10; 49J20; 49J40. 
if and only if $(X, d)$ is a geodesic space (to be defined in Section 2) and

$$
d(v,(1-t) x \oplus t y)^{p} \leq(1-t) d(v, x)^{p}+t d(v, y)^{p}-\frac{c}{2} t(1-t) d(x, y)^{p} \forall x, y, v \in X, t \in[0,1] .
$$

Let $X$ be a $p$-uniformly convex metric space. Choi and Ji [14] introduced the notion of resolvent mapping of a proper, convex and lower semicontinuous functional $f$ in $X$ as follows: For $x \in X$ and $\mu>0, J_{\mu}^{f}: X \rightarrow X$ is defined by

$$
J_{\mu}^{f}(x)=\arg \min _{v \in X}\left(f(v)+\frac{1}{2 \mu} d^{p}(v, x)\right) .
$$

Clearly, if $p=2$, then (1.3) reduces to the Moreau-Yosida resolvent. Using (1.3), they obtained the following result.

Theorem 1.1. [14, Theorem 3.6] Let $X$ be a p-uniformly convex metric space with parameter $c>0$ and diameter $\alpha>0$. Let $f: X \rightarrow(-\infty, \infty]$ be a proper uniformly convex, lower semicontinuous function, and $\left\{\mu_{n}\right\}$ be a sequence of positive real numbers such that $\lim _{n \rightarrow \infty} \frac{n}{\left(\sum_{i=1}^{n} \mu_{i}\right)}=0$. Suppose that the sequence $\left\{x_{n}\right\}$ in $X$ is generated by the following PPA:

$$
x_{n}=J_{\mu_{n}}^{f}\left(x_{n-1}\right), n \geq 1,
$$

where $J_{\mu_{n}}^{f}$ is defined in (1.3). Then, $\left\{x_{n}\right\}$ converges to a minimizer of $f$.

Kuwae [28] defined the resolvent $J_{\mu}^{f}$ of $f$ in $p$-uniformly convex metric space slightly different from that in (1.3) as follows:

$$
J_{\mu}^{f}(x)=\arg \min _{v \in X}\left(f(v)+\frac{1}{p \mu^{p-1}} d(v, x)^{p}\right) .
$$

off course, (1.5) is more general and known to be applicable in obtaining solutions of initial boundary value problems for $p$-harmonic maps (see [28] for more details). Kuwae [28] also established the unique existence of the resolvent $J_{\mu}^{f}$ of $f$ under Assumption 3.21 of [28] (see [28, Proposition 3.26]). Furthermore, he proved the existence of the minimizer of a coercive proper lower semicontinuous functionals.

We emphasize here that the results of Kuwae [28], Choi and Ji [14] naturally extend contemporary results in Hadamard spaces (as well as Hilbert and Banach spaces). For example, Theorem 1.1 is an extension of [6, Theorem 1.4] from Hadamard space to $p$-uniformly convex spaces. In general, existing results concerning PPA in Hadamard spaces cannot be simply carried into $p$-uniformly convex metric spaces due to the structure of the space; the smoothness constant $c$ (see inequality (1.2)) among others, always serves as a natural obstacle to be overcome in order to extend existing results on PPA to $p$-uniformly convex metric space. Moreover, CAT(0) spaces are 2-uniformly convex metric spaces with parameter $c=2$ and $\operatorname{CAT}(\mathrm{k})$ spaces $(k>0)$ with $\operatorname{diam}(X)<\frac{\pi}{2 \sqrt{k}}$ are 2-uniformly convex metric spaces with parameter $c=(\pi-2 \sqrt{k} \epsilon) \tan (\sqrt{k} \epsilon)$ for any $0<$ $\epsilon \leq \frac{\pi}{2 \sqrt{k}}-\operatorname{diam}(X)$ (see [42]). Furthermore, $p$-uniformly convex metric spaces are obvious generalization of $p$-uniformly convex normed spaces without using the modulus of convexity for $p \in(1, \infty)$ (see [14, Example $2.2])$. It is also known in [47] that normed spaces and their convex subsets are convex metric spaces but the converse of this statement is not always the case. In addition, inequality (1.2) has numerous applications in Finsler geometry and metric geometry; the nonlinearization of the geometry of Banach space and other related fields (see for example [29, 34, 39, 36, 37, 38, 45]). For more details on $p$-uniformly convex metric spaces see $[29,34,39,36,37,38]$ and the references therein.

Motivated by the above results and facts, we study some proximal-type algorithms for finding solutions of Split Minimization Problems (SMP) in $p$-uniformly convex metric spaces. We also study the asymptotic behaviour of the sequence generated by Halpern-type PPA and extend it to approximate a common solution of finite family of MPs in $p$-uniformly convex metric spaces. Furthermore, numerical experiments of our algorithms in comparison with other algorithms are given to show the applicability of our results

This paper is organized as follows: In section 2, we study the geometry of $p$-uniformly convex metric spaces. We also study some fundamental properties of the resolvent defined in (1.5). In section 3, we carry out strong convergence analysis on some proximal-type algorithms. First (in Subsection 3.1), we study the BackwardBackward Algorithm (BBA) and its convergence to a SMP. Secondly (in Subsection 3.2), we recall the importance of the Alternating Proximal Algorithm (APA) and prove that it converges strongly to a SMP. Finally (in Subsection 3.3), we study asymptotic behaviour of the sequence generated by Halpern-type PPA and extend it to examine the behaviour of the sequence given by Halpern-type algorithm involving a finite composition of 
resolvents of proper convex and lower semicontinuous functions. We then employ this algorithm to approximate a common solution of finite family of MPs in a complete $p$-uniformly convex metric space. In Section 4 , we give numerical example of our algorithms and compare them with other useful algorithms.

\section{Preliminaries}

\subsection{Geometry of p-uniformly convex metric space.}

Definition 2.1. A metric space $X$ is called a geodesic space if every two points $x, y \in X$ are joined by a geodesic path $c:[0, d(x, y)] \rightarrow X$ such that $c(0)=x$ and $c(d(x, y))=y$. In this case, $c$ is an isometry and the image of $c$ is called a geodesic segment joining $x$ to $y$. The space $X$ is said to be uniquely geodesic if every two points of $X$ are joined by exactly one geodesic segment.

Inequality (1.2) ensures that $p$-uniformly convex metric spaces are uniquely geodesic (see [38, Lemma 2.2]). Also, CAT(0) spaces are examples of uniquely geodesic metric spaces (see [18]). Concrete examples of $p$-uniformly convex metric spaces can be found in [14].

Definition 2.2. Let $\left\{x_{n}\right\}$ be a bounded sequence in a geodesic metric space $X$. Then, the asymptotic center $A\left(\left\{x_{n}\right\}\right)$ of $\left\{x_{n}\right\}$ is defined by

$$
A\left(\left\{x_{n}\right\}\right)=\left\{\bar{v} \in X: \limsup _{n \rightarrow \infty} d\left(\bar{v}, x_{n}\right)=\inf _{v \in X} \limsup _{n \rightarrow \infty} d\left(v, x_{n}\right) .\right.
$$

The sequence $\left\{x_{n}\right\}$ in $X$ is said to be $\Delta$-convergent to a point $\bar{v} \in X$ if $A\left(\left\{x_{n_{k}}\right\}\right)=\{\bar{v}\}$ for every subsequence $\left\{x_{n_{k}}\right\}$ of $\left\{x_{n}\right\}$. In this case, we write $\Delta-\lim _{n \rightarrow \infty} x_{n}=\bar{v}$ and we say that $\bar{v}$ is the $\Delta$-limit of $\left\{x_{n}\right\}$. The notion of $\Delta$-convergence in a metric space was introduced by Lim [31], and it is known as an analogue of the notion of weak convergence in a Banach space. Thus, it is sometimes referred to as the notion of weak convergence in metric space.

Definition 2.3. Let $X$ and $Y$ be two complete $p$-uniformly convex metric spaces. Then the Cartesian product $X \times Y$ is a complete $p$-uniformly convex metric space endowed with the metric $d:(X \times Y) \times(X \times Y) \rightarrow[0, \infty)$ defined by

$$
d\left(\left(x_{1}, y_{1}\right),\left(x_{2}, y_{2}\right)\right)=\left[d_{X}\left(x_{1}, x_{2}\right)^{p}+d_{Y}\left(y_{1}, y_{2}\right)^{p}\right]^{\frac{1}{p}}, \forall x_{1}, x_{2} \in X, y_{1}, y_{2} \in Y .
$$

The following lemma plays an important role in this paper.

Lemma 2.4. For $1<p<\infty$, let $X$ be a p-uniformly convex metric space with parameter $c>0$ and $f: X \rightarrow$ $(-\infty,+\infty]$ be a proper convex and lower semicontinuous function. Then, for all $a, b, c, d \in X$, we have

$$
d(a, b)^{p}+d(c, d)^{p} \leq \frac{2}{c}\left(d(a, c)^{p}+d(a, d)^{p}+d(b, c)^{p}+d(b, d)^{p}\right) .
$$

Proof. From (1.2), we obtain that

$$
\begin{aligned}
0 & \leq d\left(\frac{1}{2} a \oplus \frac{1}{2} b, \frac{1}{2} c \oplus \frac{1}{2} d\right)^{p} \\
& \leq \frac{1}{4}\left[d(a, c)^{p}+d(a, d)^{p}+d(b, c)^{p}+d(b, d)^{p}-\frac{c}{2}\left(d(c, d)^{p}+d(a, b)^{p}\right)\right],
\end{aligned}
$$

which implies

$$
d(a, b)^{p}+d(c, d)^{p} \leq \frac{2}{c}\left(d(a, c)^{p}+d(a, d)^{p}+d(b, c)^{p}+d(b, d)^{p}\right) .
$$

\subsection{Fundamental properties of resolvent of convex functions.}

Definition 2.5. Let $X$ be a geodesic space. A mapping $f: D \subseteq X \rightarrow(-\infty, \infty]$ is called convex if for any geodesic path $[x, y]:=\{t x \oplus(1-t) y: 0 \leq t \leq 1\}$ joining $x, y \in X$, we have that

$$
f(t x \oplus(1-t) y) \leq t f(x)+(1-t) f(y),
$$

and is called uniformly convex (see [14]), if there exists a strictly increasing function $\psi: \mathbb{R}_{+} \rightarrow \mathbb{R}_{+}$such that

$$
f\left(\frac{1}{2} x \oplus \frac{1}{2} y\right) \leq \frac{1}{2}[f(x)+f(y)]-\psi(d(x, y)) .
$$


We also recall that $f: D \subseteq X \rightarrow(-\infty, \infty]$ is called proper, if its domain $D:=\{v \in X: f(v)<+\infty\} \neq \emptyset$, and $f$ is said to be lower semi-continuous at a point $\bar{v} \in D$ if $f(\bar{v}) \leq \liminf _{n \rightarrow \infty} f\left(x_{n}\right)$ for each sequence $\left\{x_{n}\right\}$ in $D$ such that $\lim _{n \rightarrow \infty} x_{n}=\bar{v}$.

Proposition 2.6. [46] Let $X$ be a geodesic space and $f: X \rightarrow(-\infty,+\infty$ ] be a proper uniformly convex and lower semicontinuous function. Then, there exists a unique minimizer $\bar{v} \in X$ of $f$ (that is $\bar{v}:=\underset{v \in X}{\operatorname{argmin}} f(v)$ ).

Proposition 2.7. For $1<p<\infty$, let $X$ be a $p$-uniformly convex metric space with parameter $c>0$ and $f: X \rightarrow(-\infty,+\infty]$ be a proper convex and lower semicontinuous function. Then, for any $\mu>0$ and $x \in X$, there exists a unique point, say $J_{\mu}^{f}(x) \in X$ such that

$$
f\left(J_{\mu}^{f}(x)\right)+\frac{1}{p \mu^{p-1}} d\left(J_{\mu}^{f}(x), x\right)^{p}=\inf _{v \in X}\left(f(v)+\frac{1}{p \mu^{p-1}} d(v, x)^{p}\right) .
$$

Proposition 2.7 (referred to as the unique existence of resolvent of a proper convex and lower semicontinuous function) is proved in [28, Proposition 3.26] under Assumption 3.21 of [28]. Using Proposition 2.6, we prove Proposition 2.7 without this assumption. Our proof is similar to the proof of [14, Lemma 3].

Proof. Let $G_{\mu}^{f}(v):=f(v)+\frac{1}{p \mu^{p-1}} d(v, x)^{p}$. Clearly, $G_{\mu}^{f}$ is a proper and lower semicontinuous mapping. Also, $G_{\mu}^{f}$ is uniformly convex. For this, let $v=t v_{1} \oplus(1-t) v_{2}$ for all $v_{1}, v_{2} \in X$ and $t \in[0,1]$ (in particular, $t=\frac{1}{2}$ ), we obtain from the convexity of $f$ and (1.2) that

$$
\begin{aligned}
G_{\mu}^{f}\left(\frac{1}{2} v_{1} \oplus \frac{1}{2} v_{2}\right) & \leq \frac{1}{2}\left(f\left(v_{1}\right)+\frac{1}{p \mu^{p-1}} d\left(v_{1}, x\right)^{p}\right)+\frac{1}{2}\left(f\left(v_{2}\right)+\frac{1}{p \mu^{p-1}} d\left(v_{2}, x\right)^{p}\right)-\frac{c}{8 p \mu^{p-1}} d\left(v_{1}, v_{2}\right)^{p} \\
& =\frac{1}{2} G_{\mu}^{f}\left(v_{1}\right)+G_{\mu}^{f}\left(v_{2}\right)-\frac{c}{8 p \mu^{p-1}} d\left(v_{1}, v_{2}\right)^{p},
\end{aligned}
$$

which implies that $G_{\mu}^{f}$ is uniformly convex. Hence, by Proposition 2.6, we obtain the desired conclusion.

We now obtain some basic properties of the resolvent of a proper convex and lower semicontinuous function.

Lemma 2.8 (Firmly nonexpansive-type property). For $1<p<\infty$, let $X$ be a p-uniformly convex metric space with parameter $c>0$ and $f: X \rightarrow(-\infty,+\infty]$ be a proper convex and lower semicontinuous function. Then, for all $x_{1}, x_{2} \in X$, we have

$$
d\left(J_{\mu}^{f} x_{1}, J_{\mu}^{f} x_{2}\right)^{p} \leq \frac{1}{c}\left[d\left(J_{\mu}^{f} x_{1}, x_{2}\right)+d\left(J_{\mu}^{f} x_{2}, x_{1}\right)^{p}-d\left(J_{\mu}^{f} x_{1}, x_{1}\right)^{p}-d\left(J_{\mu}^{f} x_{2}, x_{2}\right)^{p}\right] .
$$

Proof. From (1.5), we obtain that

$$
f\left(J_{\mu}^{f} x\right)+\frac{1}{p \mu^{p-1}} d\left(J_{\mu}^{f} x, x\right)^{p} \leq f(z)+\frac{1}{p \mu^{p-1}} d(z, x)^{p} \forall z \in X .
$$

Now, set $z=(1-t) v \oplus t J_{\mu}^{f} x, t \in[0,1)$. Then, we obtain from the convexity of $f$ and the inequality (1.2) that

$$
\begin{aligned}
f\left(J_{\mu}^{f} x\right)+\frac{1}{p \mu^{p-1}} d\left(J_{\mu}^{f} x, x\right)^{p} \leq & (1-t) f(v)+t f\left(J_{\mu}^{f} x\right)+\frac{(1-t)}{p \mu^{p-1}} d(v, x)^{p} \\
& +\frac{t}{p \mu^{p-1}} d\left(J_{\mu}^{f} x, x\right)^{p}-\frac{c t(1-t)}{2 p \mu^{p-1}} d\left(v, J_{\mu}^{f} x\right)^{p},
\end{aligned}
$$

which implies (since $t \neq 1$ ) that

$$
p \mu^{p-1} f\left(J_{\mu}^{f} x\right)+d\left(J_{\mu}^{f} x, x\right)^{p} \leq p \mu^{p-1} f(v)+d(v, x)^{p}-\frac{c t}{2} d\left(v, J_{\mu}^{f} x\right)^{p} .
$$

As $t \rightarrow 1$ in (2.2), we obtain

$$
p \mu^{p-1} f\left(J_{\mu}^{f} x\right)+d\left(J_{\mu}^{f} x, x\right)^{p} \leq p \mu^{p-1} f(v)+d(v, x)^{p}-\frac{c}{2} d\left(v, J_{\mu}^{f} x\right)^{p} .
$$

Now, for $x_{1}, x_{2} \in X$, we obtain from (2.3) that

$$
p \mu^{p-1} f\left(J_{\mu}^{f} x_{1}\right)+d\left(J_{\mu}^{f} x_{1}, x_{1}\right)^{p} \leq p \mu^{p-1} f\left(J_{\mu}^{f} x_{2}\right)+d\left(J_{\mu}^{f} x_{2}, x_{1}\right)^{p}-\frac{c}{2} d\left(J_{\mu}^{f} x_{2}, J_{\mu}^{f} x_{1}\right)^{p}
$$

and

$$
p \mu^{p-1} f\left(J_{\mu}^{f} x_{2}\right)+d\left(J_{\mu}^{f} x_{2}, x_{2}\right)^{p} \leq p \mu^{p-1} f\left(J_{\mu}^{f} x_{1}\right)+d\left(J_{\mu}^{f} x_{1}, x_{2}\right)^{p}-\frac{c}{2} d\left(J_{\mu}^{f} x_{1}, J_{\mu}^{f} x_{2}\right)^{p} .
$$


Adding (2.4) and (2.5), we obtain

$$
d\left(J_{\mu}^{f} x_{1}, J_{\mu}^{f} x_{2}\right)^{p} \leq \frac{1}{c}\left[d\left(J_{\mu}^{f} x_{1}, x_{2}\right)+d\left(J_{\mu}^{f} x_{2}, x_{1}\right)^{p}-d\left(J_{\mu}^{f} x_{1}, x_{1}\right)^{p}-d\left(J_{\mu}^{f} x_{2}, x_{2}\right)^{p}\right] .
$$

Remark 2.9. (a) Observe that if $c \geq 2$ and $p=2$ in Lemma 2.8, then by the definition of quasilinearization mapping in $\mathrm{CAT}(0)$ space (see [11] and [49]), one obtains that $J_{\mu}^{f}$ is a firmly nonexpansive mapping in a $\operatorname{CAT}(0)$ space. That is,

$$
d\left(J_{\mu}^{f} x_{1}, J_{\mu}^{f} x_{2}\right)^{2} \leq\left\langle\overrightarrow{J_{\mu}^{f} x_{1} J_{\mu}^{f} x_{2}}, \overrightarrow{x_{1} x_{2}}\right\rangle \forall x_{1}, x_{2} \in X,
$$

which by Cauchy-Swartz inequality gives that $J_{\mu}^{f}$ is nonexpansive in $\operatorname{CAT}(0)$ space.

(b) From (2.3), we obtain that

$$
d\left(v, J_{\mu}^{f} x\right)^{p} \leq \frac{2}{c}\left[d(v, x)^{p}-d\left(J_{\mu}^{f} x, x\right)^{p}-p \mu^{p-1}\left(f\left(J_{\mu}^{f} x\right)-f(v)\right)\right], \forall v \in X .
$$

(c) If we replace convexity of $f$ with uniform convexity in Lemma 2.8, then (b) becomes

$$
d\left(v, J_{\mu}^{f} x\right)^{p} \leq \frac{2}{c}\left[d(v, x)^{p}-d^{p}\left(J_{\mu}^{f} x, x\right)-p \mu^{p-1}\left(\psi\left(d\left(v, J_{\mu}^{f} x\right)\right)+f\left(J_{\mu}^{f} x\right)-f(v)\right)\right], \forall v \in X .
$$

Lemma 2.10 (Nonexpansive property). For $1<p<\infty$, let $X$ be a p-uniformly convex metric space with parameter $c \geq 2$ and $f: X \rightarrow(-\infty,+\infty]$ be a proper convex and lower semicontinuous function. Then, the resolvent $J_{\mu}^{f}$ of $f$ is nonexpansive. That is, for all $x_{1}, x_{2} \in X$, we have

$$
d\left(J_{\mu}^{f} x_{1}, J_{\mu}^{f} x_{2}\right) \leq d\left(x_{1}, x_{2}\right) .
$$

Proof. By Lemma 2.4 and Lemma 2.8 (note that $c \geq 2$ ), we obtain that

$$
\begin{aligned}
d\left(J_{\mu}^{f} x_{1}, J_{\mu}^{f} x_{2}\right)^{p} & \leq \frac{1}{c}\left[\frac{2}{c}\left(d\left(J_{\mu}^{f} x_{1}, J_{\mu}^{f} x_{2}\right)^{p}+d\left(J_{\mu}^{f} x_{1}, x_{1}\right)+d\left(J_{\mu}^{f} x_{2}, x_{2}\right)^{p}+d\left(x_{1}, x_{2}\right)^{p}\right)-d\left(J_{\mu}^{f} x_{1}, x_{1}\right)^{p}-d\left(J_{\mu}^{f} x_{2}, x_{2}\right)^{p}\right] \\
& \leq \frac{1}{2}\left[d\left(J_{\mu}^{f} x_{1}, J_{\mu}^{f} x_{2}\right)^{p}+d\left(x_{1}, x_{2}\right)^{p}\right],
\end{aligned}
$$

which yields the desired conclusion.

Lemma 2.11 (Monotonicity of resolvent). For $1<p<\infty$, let $X$ be a p-uniformly convex metric space with parameter $c>0$ and $f: X \rightarrow(-\infty,+\infty]$ be a proper convex and lower semicontinuous function. Then, for $0<\mu_{1}<\mu_{2}$, we have

$$
d\left(J_{\mu_{1}}^{f} x, x\right) \leq d\left(J_{\mu_{2}}^{f} x, x\right) \forall x \in X .
$$

Proof. Let $x \in X$. We obtain from (1.5) that

$$
f\left(J_{\mu_{2}}^{f} x\right)+\frac{1}{p \mu_{2}^{p-1}} d\left(J_{\mu_{2}}^{f} x, x\right)^{p} \leq f\left(J_{\mu_{1}}^{f} x\right)+\frac{1}{p \mu_{2}^{p-1}} d\left(J_{\mu_{1}}^{f} x, x\right)^{p} .
$$

Similarly, we obtain

$$
f\left(J_{\mu_{1}}^{f} x\right)+\frac{1}{p \mu_{1}^{p-1}} d\left(J_{\mu_{1}}^{f} x, x\right)^{p} \leq f\left(J_{\mu_{2}}^{f} x\right)+\frac{1}{p \mu_{1}^{p-1}} d\left(J_{\mu_{2}}^{f} x, x\right)^{p} .
$$

Adding (2.6) and (2.7), we obtain that

$$
\left(1-\frac{\mu_{1}^{p-1}}{\mu_{2}^{p-1}}\right) d\left(J_{\mu_{1}}^{f} x, x\right)^{p} \leq\left(1-\frac{\mu_{1}^{p-1}}{\mu_{2}^{p-1}}\right) d\left(J_{\mu_{2}}^{f} x, x\right)^{p} .
$$

Since, $0<\mu_{1}<\mu_{2}$, therefore $1-\left(\frac{\mu_{1}}{\mu_{2}}\right)^{p-1}>0$. Thus, we obtain that

$$
d\left(J_{\mu_{1}}^{f} x, x\right) \leq d\left(J_{\mu_{2}}^{f} x, x\right) .
$$

We end this section with the following important result which is an analogue of [27, Lemma 3.1] in the setting of $\operatorname{CAT}(0)$ space. 
Lemma 2.12. For $1<p<\infty$, let $X$ be a p-uniformly convex metric space with parameter $c>0$ and $f: X \rightarrow$ $(-\infty,+\infty]$ be a proper, convex and lower semicontinuous function. For $\mu_{1}, \mu_{2}>0$ and $x_{1}, x_{2} \in X$, the following inequality holds:

$\frac{c}{2}\left(\mu_{1}^{p-1}+\mu_{2}^{p-1}\right) d\left(J_{\mu_{1}}^{f} x_{1}, J_{\mu_{2}}^{f} x_{2}\right)^{p}+\mu_{2}^{p-1} d\left(J_{\mu_{1}}^{f} x_{1}, x_{1}\right)^{p}+\mu_{1}^{p-1} d\left(J_{\mu_{2}}^{f} x_{2}, x_{2}\right)^{p} \leq \mu_{1}^{p-1} d\left(J_{\mu_{1}}^{f} x_{1}, x_{2}\right)^{p}+\mu_{2}^{p-1} d\left(J_{\mu_{2}}^{f} x_{2}, x_{1}\right)^{p}$.

Proof. Put $x=x_{1}$ and $v=J_{\mu_{2}}^{f} x_{2}$ in (2.3) to obtain

$$
p \mu_{1}{ }^{p-1} f\left(J_{\mu_{1}}^{f} x_{1}\right)+d\left(J_{\mu_{1}}^{f} x_{1}, x_{1}\right)^{p} \leq p \mu_{1}^{p-1} f\left(J_{\mu_{2}}^{f} x_{2}\right)+d\left(J_{\mu_{2}}^{f} x_{2}, x_{1}\right)^{p}-\frac{c}{2} d\left(J_{\mu_{2}} x_{2}, J_{\mu_{1}}^{f} x_{1}\right)^{p} .
$$

That is,

$$
\frac{c}{2} d\left(J_{\mu_{1}}^{f} x_{1}, J_{\mu_{2}}^{f} x_{2}\right)^{p}+d\left(J_{\mu_{1}}^{f} x_{1}, x_{1}\right)^{p}+p \mu_{1}^{p-1}\left(f\left(J_{\mu_{1}}^{f} x_{1}\right)-f\left(J_{\mu_{2}}^{f} x_{2}\right)\right) \leq d\left(J_{\mu_{2}}^{f} x_{2}, x_{1}\right)^{p},
$$

from which we obtain that

$$
\begin{aligned}
& p \mu_{2}^{p-1}\left[\frac{c}{2} d\left(J_{\mu_{1}}^{f} x_{1}, J_{\mu_{2}}^{f} x_{2}\right)^{p}+d\left(J_{\mu_{1}}^{f} x_{1}, x_{1}\right)^{p}+p \mu_{1}^{p-1}\left(f\left(J_{\mu_{1}}^{f} x_{1}\right)-f\left(J_{\mu_{2}}^{f} x_{2}\right)\right)\right] \\
& \leq p \mu_{2}^{p-1} d\left(J_{\mu_{2}}^{f} x_{2}, x_{1}\right)^{p} .
\end{aligned}
$$

Similarly, we obtain

$$
\begin{aligned}
& p \mu_{1}^{p-1}\left[\frac{c}{2} d\left(J_{\mu_{2}}^{f} x_{2}, J_{\mu_{1}}^{f} x_{1}\right)^{p}+d\left(J_{\mu_{2}}^{f} x_{2}, x_{2}\right)^{p}+p \mu_{2}^{p-1}\left(f\left(J_{\mu_{2}}^{f} x_{2}\right)-f\left(J_{\mu_{1}}^{f} x_{1}\right)\right)\right] \\
& \leq p \mu_{1}^{p-1} d\left(J_{\mu_{1}}^{f} x_{1}, x_{2}\right)^{p} .
\end{aligned}
$$

Adding (2.8) and (2.9), we obtain the desired conclusion.

\section{Strong CONVERGEnCE ANALYsis}

In this section, we study strong convergence of some proximal-type algorithms.

Remark 3.1. In general, PPA is known to converge only weakly even in a Hilbert space; to obtain strong convergence results for PPA (see [6]), we need to impose additional assumption(s) on either the convex function or on the underlying space.

Since our interest in this paper is to obtain strong convergence results, we shall rely on the above remark in our study. That is, we shall assume in the next two subsections that, the proper lower semicontinuous function $f$ is uniformly convex, and in the last subsection that, the smoothness constant $c$ of $X$ is in $[2, \infty$ ) (in this case, $f$ needs not to be uniformly convex).

3.1. Backward-backward algorithm. The BBA is defined for an initial point $x_{1} \in X$ as:

$$
\left\{\begin{array}{l}
y_{n}=J_{\mu_{n}}^{g} x_{n}, \\
x_{n+1}=J_{\mu_{n}}^{f} y_{n}, n \geq 1,
\end{array}\right.
$$

where $\left\{\mu_{n}\right\}$ is a sequence of positive real numbers and $f, g: X \rightarrow(-\infty, \infty]$ are two proper, convex and lower semicontinuous functions (see [8] for related work in the frame work of Hadamard spaces). In what follows, we shall study strong convergence of Algorithm (3.1) to a solution of the following SMP:

$$
\min \Psi(x, y) \text { such that }(x, y) \in X \times X \text {, where } \Psi(x, y)=f(x)+g(y) \forall x, y \in X \text {. }
$$

We begin with the following lemma.

Lemma 3.2. For $1<p<\infty$, let $X$ be a p-uniformly convex metric space with parameter $c>0$ and $f, g: X \rightarrow$ $(-\infty,+\infty]$ be two proper, convex and lower semicontinuous functions. Let $\left\{x_{n}\right\}$ and $\left\{y_{n}\right\}$ be defined by (3.1), where $\left\{\mu_{n}\right\}$ is a sequence of positive real numbers. Then, for any $v=(x, y) \in X \times X$, we have

$$
\Psi\left(v_{n}\right)-\Psi(v) \leq \frac{\sum_{i=1}^{n-1} d\left(v, v_{i}\right)^{p}-\frac{c}{2} \sum_{i=2}^{n} d\left(v, v_{i}\right)^{p}}{p \sum_{i=1}^{n-1} \mu_{i}^{p-1}},
$$

where $v_{n}=\left(x_{n}, y_{n}\right) \in X \times X$. 
Proof. By (3.1) and (1.5), we obtain that

$$
g\left(y_{n}\right)+\frac{1}{p \mu_{n}^{p-1}} d\left(y_{n}, x_{n}\right)^{p} \leq g(y)+\frac{1}{p \mu_{n}^{p-1}} d\left(x_{n}, y\right)^{p}
$$

and

$$
f\left(x_{n+1}\right)+\frac{1}{p \mu_{n}^{p-1}} d\left(x_{n+1}, y_{n}\right)^{p} \leq f(x)+\frac{1}{p \mu_{n}^{p-1}} d\left(y_{n}, x\right)^{p}
$$

Adding (3.4) and (3.5), we obtain for all $x, y \in X$ that

$$
\begin{aligned}
f\left(x_{n+1}\right)+g\left(y_{n}\right)+\frac{1}{p \mu_{n}^{p-1}}\left[d\left(x_{n+1}, y_{n}\right)^{p}+d\left(y_{n}, x_{n}\right)^{p}\right] \leq & f(x)+g(y) \\
& +\frac{1}{p \mu_{n}^{p-1}}\left[d\left(y_{n}, x\right)^{p}+d\left(x_{n}, y\right)^{p}\right] .
\end{aligned}
$$

In particular, for $y=y_{n}$, we obtain that

$$
f\left(x_{n+1}\right)+\frac{1}{p \mu_{n}^{p-1}}\left[d\left(x_{n+1}, y_{n}\right)^{p}+d\left(y_{n}, x_{n}\right)^{p}\right] \leq f(x)+\frac{1}{p \mu_{n}^{p-1}}\left[d\left(y_{n}, x\right)^{p}+d\left(x_{n}, y_{n}\right)^{p}\right] .
$$

Now, by interchanging $f$ and $g$, and starting the iteration process at $y_{1}$ in (3.1), then by an argument similar to above, we obtain that

$$
\begin{aligned}
g\left(y_{n+1}\right)+f\left(x_{n}\right)+\frac{1}{p \mu_{n}^{p-1}}\left[d\left(y_{n+1}, x_{n}\right)^{p}+d\left(x_{n}, y_{n}\right)^{p}\right] \leq & g(y)+f(x) \\
& +\frac{1}{p \mu_{n}^{p-1}}\left[d\left(x_{n}, y\right)^{p}+d\left(y_{n}, x\right)^{p}\right] .
\end{aligned}
$$

By setting $x=x_{n}$ in (3.8), we obtain

$$
g\left(y_{n+1}\right)+\frac{1}{p \mu_{n}^{p-1}}\left[d\left(y_{n+1}, x_{n}\right)^{p}+d\left(x_{n}, y_{n}\right)^{p}\right] \leq g(y)+\frac{1}{p \mu_{n}^{p-1}}\left[d\left(x_{n}, y\right)^{p}+d\left(y_{n}, x_{n}\right)^{p}\right] .
$$

Adding (3.7) and (3.9), we obtain

$$
\begin{aligned}
f\left(x_{n+1}\right)+g\left(y_{n+1}\right)+\frac{1}{p \mu_{n}^{p-1}}\left[d\left(x_{n+1}, y_{n}\right)^{p}+d\left(y_{n+1}, x_{n}\right)^{p}\right] \leq & f(x)+g(y) \\
& +\frac{1}{p \mu_{n}^{p-1}}\left[d\left(x_{n}, y\right)^{p}+d\left(y_{n}, x\right)^{p}\right],
\end{aligned}
$$

which gives by (2.1) that

$$
\Psi\left(v_{n+1}\right)+\frac{1}{p \mu_{n}^{p-1}} d\left(v_{n+1}, v_{n}\right)^{p} \leq \Psi(v)+\frac{1}{p \mu_{n}^{p-1}} d\left(v_{n}, v\right)^{p} .
$$

Thus, by Remark 2.9 (b), (or inequality (2.3)), we obtain that

$$
p \mu_{n}^{p-1}\left(\Psi\left(v_{n+1}\right)-\Psi(v)\right) \leq d\left(v, v_{n}\right)^{p}-\frac{c}{2} d\left(v, v_{n+1}\right)^{p} .
$$

By letting $v=v_{n}$ in (3.10), we obtain that

$$
\Psi\left(v_{n+1}\right)+\frac{1}{p \mu_{n}^{p-1}} d\left(v_{n+1}, v_{n}\right)^{p} \leq \Psi\left(v_{n}\right),
$$

which implies that $\left\{\Psi\left(v_{n}\right)\right\}$ is monotone non-increasing. Thus, we obtain from (3.11) that

$$
\begin{aligned}
p\left(\Psi\left(v_{n}\right)-\Psi(v)\right) \sum_{i=1}^{n-1} \mu_{i}^{p-1} & \leq p \sum_{i=1}^{n-1} \mu_{i}^{p-1}\left(\Psi\left(v_{i+1}\right)-\Psi(v)\right) \\
& \leq \sum_{i=1}^{n-1} d\left(v, v_{i}\right)^{p}-\frac{c}{2} \sum_{i=2}^{n} d\left(v, v_{i}\right)^{p},
\end{aligned}
$$

which yields the desired conclusion.

Theorem 3.3. For $1<p<\infty$, let $X$ be a complete $p$-uniformly convex metric space with parameter $c>0$ such that the diameter of $X \times X$ is $K>0$. Let $f, g: X \rightarrow(-\infty,+\infty]$ be two proper, uniformly convex and lower semicontinuous functions and $\left\{x_{n}\right\},\left\{y_{n}\right\}$ be sequences defined by (3.1), where $\left\{\mu_{n}\right\}$ is a sequence of positive real numbers such that $\lim _{n \rightarrow \infty} \frac{n}{\sum_{i=1}^{n} \mu_{i}^{p-1}}=0$. Then, $\left\{\left(x_{n}, y_{n}\right)\right\}$ converges to a solution of (3.2). 
Proof. Since the diameter of $X \times X$ is $K>0$, therefore we obtain from (3.3) that

$$
\begin{aligned}
\Psi\left(v_{n}\right)-\Psi(v) & \leq \frac{\sum_{i=1}^{n-1} d\left(v, v_{i}\right)^{p}-\frac{c}{2} \sum_{i=2}^{n} d\left(v, v_{i}\right)^{p}}{p \sum_{i=1}^{n-1} \mu_{i}^{p-1}} \\
& \leq \frac{(n-1) K^{p}}{p \sum_{i=1}^{n-1} \mu_{i}^{p-1}} \rightarrow 0, \text { as } n \rightarrow \infty .
\end{aligned}
$$

That is, $\lim _{n \rightarrow \infty} \Psi\left(v_{n}\right) \leq \Psi(v)$ for all $v \in X \times X$, which implies that

$$
\lim _{n \rightarrow \infty} \Psi\left(v_{n}\right)=\inf _{v \in(X \times X)} \Psi(v) .
$$

Furthermore, we obtain by Proposition 2.6 that, there exists a unique minimizer $\bar{v} \in(X \times X)$ of $\Psi$. Thus, by (3.14), we obtain that

$$
\lim _{n \rightarrow \infty} \Psi\left(v_{n}\right)=\Psi(\bar{v})
$$

Also, using the uniform convexity of $\Psi$, we obtain that there exists a function $\psi: \mathbb{R}_{+} \rightarrow \mathbb{R}_{+}$with $\psi(t)=0 \Longleftrightarrow$ $t=0$ such that

$$
\Psi\left(\frac{1}{2} v_{n} \oplus \frac{1}{2} v_{m}\right) \leq \frac{1}{2}\left(\Psi\left(v_{n}\right)+\Psi\left(v_{m}\right)\right)-\psi\left(d\left(v_{n}, v_{m}\right)\right), \forall n, m \geq 1 .
$$

Since $\psi(t)=0 \Longleftrightarrow t=0$, we obtain from (3.15) that $d\left(v_{n}, v_{m}\right) \rightarrow 0$, as $n, m \rightarrow \infty$. Thus, $\left\{v_{n}\right\}$ is a Cauchy sequence in $X \times X$. As $X$ is complete, so $X \times X$ is also complete. Thus, $\left\{v_{n}\right\}$ converges to a point say $\hat{v} \in X \times X$. It follows from the lower semicontinuity of $\Psi$ (since $f$ and $g$ are lower semicontinuous functions) and (3.3) that $\Psi(\hat{v})=\inf _{v \in X \times X} \Psi(v)$. Therefore, we conclude that $\left\{v_{n}\right\}=\left\{\left(x_{n}, y_{n}\right)\right\}$ converges to a solution of (3.2).

Remark 3.4. If $X$ is a complete 2 -uniformly convex metric space in Theorem 3.3 with parameter $c=2$ for $X \times X$, then (3.13) becomes

$$
\begin{aligned}
\Psi\left(v_{n}\right)-\Psi(v) & \leq \frac{\sum_{i=1}^{n-1} d\left(v, v_{i}\right)^{2}-\sum_{i=2}^{n} d\left(v, v_{i}\right)^{2}}{2 \sum_{i=1}^{n-1} \mu_{i}} \\
& \leq \frac{d\left(v, v_{1}\right)^{2}}{2 \sum_{i=1}^{n-1} \mu_{i}},
\end{aligned}
$$

which implies that $\lim _{n \rightarrow \infty} \Psi\left(v_{n}\right)=\inf _{v \in(X \times X)} \Psi(v)$, provided $\lim _{n \rightarrow \infty} \sum_{i=1}^{n-1} \mu_{i}=\infty$. In this case, we do not need the assumption that $X \times X$ has a diameter $K>0$. Thus, we obtain the following result from Theorem 3.3.

Corollary 3.5. Let $X$ be a complete 2-uniformly convex metric space (in particular, a complete CAT(0) space) and $f, g: X \rightarrow(-\infty,+\infty]$ be two proper, uniformly convex and lower semicontinuous functions. Suppose that $\left\{x_{n}\right\}$ and $\left\{y_{n}\right\}$ are sequences defined by (3.1), where $\left\{\mu_{n}\right\}$ is a sequence of positive real numbers such that $\sum_{n=1}^{\infty} \mu_{n}=\infty$. Then, $\left\{\left(x_{n}, y_{n}\right)\right\}$ converges to a solution of (3.2).

3.2. Alternating proximal algorithm. In problem (3.2), the functions $f$ and $g$ are defined on the same space $X$. In this subsection, we shall consider the SMP for the case where $f$ and $g$ are defined on two different $p$-uniformly convex metric spaces, say $X$ and $Y$ respectively. That is, we consider the following SMP:

$$
\min \Psi(x, y) \text { such that }(x, y) \in X \times Y \text {, }
$$

where $X$ and $Y$ are $p$-uniformly convex metric spaces and $\Psi: X \times Y \rightarrow(-\infty,+\infty]$ is a function defined by $\Psi(x, y)=f(x)+g(y) ; f: X \rightarrow(-\infty,+\infty]$ and $g: Y \rightarrow(-\infty,+\infty]$ are two proper convex and lower semicontinuous functions.

To solve problem (3.16), we define the following algorithm called the APA: For arbitrary point $v_{1}=\left(x_{1}, y_{1}\right)$ in $X \times Y$, the sequence $\left\{v_{n}\right\}=\left\{\left(x_{n}, y_{n}\right)\right\}$ in $X \times Y$ is defined as follows:

$$
\begin{aligned}
\left(x_{n}, y_{n}\right) \rightarrow\left(x_{n+1}, y_{n}\right) \rightarrow\left(x_{n+1}, y_{n+1}\right), & \left\{\begin{array}{l}
x_{n+1}=\underset{x \in X}{\operatorname{argmin}}\left(\Psi\left(x, y_{n}\right)+\frac{1}{p \mu_{n}^{p-1}} d\left(x_{n}, x\right)^{p}\right), x \in X, \\
y_{n+1}=\underset{y \in Y}{\operatorname{argmin}}\left(\Psi\left(x_{n+1}, y\right)+\frac{1}{p \mu_{n}^{p-1}} d\left(y_{n}, y\right)^{p}\right), y \in Y, n \geq 1,
\end{array}\right.
\end{aligned}
$$


where $\left\{\mu_{n}\right\}$ is a sequence of positive numbers. We remark here that, in each iteration, we have to solve the following subproblems:

$$
\min \Psi\left(x, y_{n}\right)+\frac{1}{p \mu_{n}^{p-1}} d^{2}\left(x_{n}, x\right), \text { where } x \in X
$$

and

$$
\min \Psi\left(x_{n+1}, y\right)+\frac{1}{p \mu_{n}^{p-1}} d^{2}\left(y_{n}, y\right), \text { where } y \in Y .
$$

In order to solve the subproblem (3.18) or (3.19), we employ the following PPA: For arbitrary $x_{1} \in X,\left\{x_{n}\right\}$ is generated by

$$
x_{n+1}=\arg \min _{x \in X}\left(f(x)+\frac{1}{p \mu_{n}^{p-1}} d\left(x_{n}, x\right)^{p}\right), n \geq 1,
$$

where $f(x)=\Psi\left(x, y_{n}\right)$. This process has been studied in several settings. For instance, in Euclidean spaces (see $[1,3]$ ), Hilbert spaces (see [2, 13]), Hadamard manifolds (see [16]) and Hadamard spaces (see [15]).

Algorithm (3.17) has many applications, for instance, it has applications in decision science ([1]), game theory $([2,16])$, PDE's and many other disciplines (see [2, 15]). Furthermore, unlike Algorithm (3.1), Algorithm (3.17) allows us to check or monitor what happens in each space of action after a given iteration (see [15]).

Therefore, it is of practical importance to study problems of the form (3.16) using Algorithm (3.17). To this end, we present the following convergence result for problem (3.16).

Theorem 3.6. For $1<p<\infty$, let $X$ and $Y$ be two complete $p$-uniformly convex metric spaces with parameter $c>0$ and such that the diameter of $X \times Y$ is $K>0$. Let $f: X \rightarrow(-\infty,+\infty]$ and $g: Y \rightarrow(-\infty,+\infty]$ be two proper, uniformly convex and lower semicontinuous functions and $\left\{\left(x_{n}, y_{n}\right)\right\}$ be the sequence defined by (3.17), where $\left\{\mu_{n}\right\}$ is a sequence of positive real numbers such that $\lim _{n \rightarrow \infty} \frac{n}{\sum_{i=1}^{n} \mu_{i}^{p-1}}=0$. Then, $\left\{\left(x_{n}, y_{n}\right)\right\}$ converges to a solution of (3.16).

Proof. By (3.17) (also see (3.20)), we obtain that

$$
f\left(x_{n+1}\right)+g\left(y_{n}\right)+\frac{1}{p \mu^{p-1}} d\left(x_{n}, x_{n+1}\right)^{p} \leq f(x)+g\left(y_{n}\right)+\frac{1}{p \mu^{p-1}} d\left(x_{n}, x\right)^{p}
$$

and

$$
g\left(y_{n+1}\right)+f\left(x_{n+1}\right)+\frac{1}{p \mu^{p-1}} d\left(y_{n}, y_{n+1}\right)^{p} \leq g(x)+f\left(x_{n+1}\right)+\frac{1}{p \mu^{p-1}} d\left(y_{n}, y\right)^{p} .
$$

Adding above two inequalities, we obtain that

$$
f\left(x_{n+1}\right)+g\left(y_{n+1}\right)+\frac{1}{p \mu^{p-1}}\left[d\left(x_{n}, x_{n+1}\right)^{p}+d\left(y_{n}, y_{n+1}\right)^{p}\right] \leq f(x)+g(y)+\frac{1}{p \mu^{p-1}}\left[d\left(x_{n}, x\right)^{p}+d\left(y_{n}, y\right)^{p}\right],
$$

which gives by (2.1) that

$$
\Psi\left(x_{n+1}, y_{n+1}\right)+\frac{1}{p \mu_{n}^{p-1}} d\left(\left(x_{n}, y_{n}\right),\left(x_{n+1}, y_{n+1}\right)\right)^{p} \leq \Psi(x, y)+\frac{1}{p \mu_{n}^{p-1}} d\left(\left(x_{n}, y_{n}\right),(x, y)\right)^{p} .
$$

Set $v=(x, y)$ and $v_{n}=\left(x_{n}, y_{n}\right)$ in $(3.23)$, to get

$$
\Psi\left(v_{n+1}\right)+\frac{1}{p \mu_{n}^{p-1}} d\left(v_{n}, v_{n+1}\right)^{p} \leq \Psi(v)+\frac{1}{p \mu_{n}^{p-1}} d\left(v_{n}, v\right)^{p} .
$$

As in the proof of (3.10) -(3.12), we can show that that

$$
\Psi\left(v_{n}\right)-\Psi(v) \leq \frac{\sum_{i=1}^{n-1} d\left(v, v_{i}\right)^{p}-\frac{c}{2} \sum_{i=2}^{n} d\left(v, v_{i}\right)^{p}}{p \sum_{i=1}^{n-1} \mu_{i}^{p-1}} .
$$

Hence, by a proof similar to that of Theorem 3.3, we obtain the desired conclusion.

Corollary 3.7. Let $X$ and $Y$ be two complete 2-uniformly convex metric spaces (in particular, complete CAT(0) spaces). Let $f: X \rightarrow(-\infty,+\infty]$ and $g: Y \rightarrow(-\infty,+\infty]$ be two proper, uniformly convex and lower semicontinuous functions. Suppose that $\left\{\left(x_{n}, y_{n}\right)\right\}$ is a sequence defined by (3.17), where $\left\{\mu_{n}\right\}$ is a sequence of positive real numbers such that $\sum_{n=1}^{\infty} \mu_{n}=\infty$. Then, $\left\{\left(x_{n}, y_{n}\right)\right\}$ converges to a solution of (3.16).

Proof. It follows from Theorem 3.6 and Remark 3.4. 
3.3. Halpern-type proximal point algorithm. In this subsection, we study the asymptotic behaviour of the sequence $\left\{x_{n}\right\}$ generated by the following Halpern-type PPA:

$$
\left\{\begin{array}{l}
u, x_{1} \in X, \\
x_{n+1}=\alpha_{n} u \oplus\left(1-\alpha_{n}\right) J_{\mu_{n}}^{f} x_{n},
\end{array}\right.
$$

where $\left\{\alpha_{n}\right\}$ and $\left\{\mu_{n}\right\}$ are sequences in $[0,1)$ and $(0, \infty)$ respectively, and $f: X \rightarrow(-\infty,+\infty]$ is a proper convex and lower semicontious function. We also extend our study to examine the behaviour of the sequence given by the following Halpern-type PPA involving finite composition of resolvents of proper convex and lower semicontinuous functions:

$$
\left\{\begin{array}{l}
u, x_{1} \in X \\
x_{n+1}=\alpha_{n} u \oplus\left(1-\alpha_{n}\right) \prod_{j=1}^{m} J_{\mu_{n}}^{f_{j}} x_{n}, n \geq 1,
\end{array}\right.
$$

where $\prod_{j=1}^{m} J_{\mu_{n}}^{f_{j}}=J_{\mu_{n}}^{f_{1}} \circ J_{\mu_{n}}^{f_{2}} \circ \cdots \circ J_{\mu_{n}}^{f_{m-1}} \circ J_{\mu_{n}}^{f_{m}},\left\{\alpha_{n}\right\}$ is a sequence in $[0,1)$ and $\left\{\mu_{n}\right\}$ is a sequence in $(0, \infty)$. We shall employ Algorithm (3.27) to find common solution of a finite family of MPs.

For our strong convergence results in this subsection, we only need the proper lower semicontinuous function $f$ to be convex (not uniformly convex). However, we shall assume that the smoothness constant $c$ of the $p$-uniformly convex metric space $X$ is in $[2, \infty)$ (see Remark 3.1).

Lemma 3.8. For $1<p<\infty$, let $X$ be a p-uniformly convex metric space with parameter $c \geq 2$ and $f: X \rightarrow$ $(-\infty,+\infty]$ be proper, convex and lower semicontinuous function such that for $\mu>0 F\left(J_{\mu}^{f}\right) \neq \emptyset$ (where $F\left(J_{\mu}^{f}\right)$ denotes the set of fixed points of $\left.J_{\mu}^{f}\right)$. Then, $F\left(J_{\mu}^{f}\right)=\underset{y \in X}{\operatorname{argmin}} f(y)$.

Proof. Let $\bar{v} \in F\left(J_{\mu}^{f}\right)$. Then, by (1.5), we obtain that

$$
f(\bar{v}) \leq f(v)+\frac{1}{p \mu^{p-1}} d(v, \bar{v})^{p} .
$$

Let $v=(1-t) y \oplus t \bar{v}$ for all $y \in X$ and $t \in[0,1)$. Then, by the convexity of $f$ and (1.2), we obtain that

$$
(1-t) f(\bar{v}) \leq(1-t) f(y)+\frac{(1-t)}{p \mu^{p-1}} d(y, \bar{v})^{p}+\frac{t}{p \mu^{p-1}} d(\bar{v}, \bar{v})^{p}-\frac{c t(1-t)}{2 p \mu^{p-1}} d(y, \bar{v})^{p} .
$$

Since $c \geq 2$, therefore we obtain that

$$
\frac{t(1-t)}{p \mu^{p-1}} d(y, \bar{v})^{p} \leq(1-t)(f(y)-f(\bar{v}))+\frac{(1-t)}{p \mu^{p-1}} d(y, \bar{v})^{p},
$$

which implies that

As $t \rightarrow 1$, we obtain that

$$
t d(y, \bar{v})^{p} \leq p \mu^{p-1}(f(y)-f(\bar{v}))+d(y, \bar{v})^{p} .
$$

Hence, $\bar{v} \in \underset{y \in X}{\operatorname{argmin}} f(y)$.

$$
0 \leq f(y)-f(\bar{v}) \forall y \in X
$$

Conversely, suppose that $\bar{v} \in \underset{y \in X}{\operatorname{argmin}} f(y)$. Then, we obtain by (1.5) that

$$
f\left(J_{\mu}^{f} \bar{v}\right)+\frac{1}{p \mu^{p-1}} d\left(J_{\mu}^{f} \bar{v}, \bar{v}\right)^{p} \leq f(v)+\frac{1}{p \mu^{p-1}} d(v, \bar{v})^{p} .
$$

Let $v=(1-t) \bar{v} \oplus t J_{\mu}^{f} \bar{v}$, for $t \in[0,1)$. Then, we obtain by the convexity of $f$ and (1.2) that

$$
\begin{aligned}
\frac{1}{p \mu^{p-1}} d\left(J_{\mu}^{f} \bar{v}, \bar{v}\right)^{p} & \leq(1-t) f(\bar{v})-(1-t) f\left(J_{\mu}^{f} \bar{v}\right)+\frac{1}{p \mu^{p-1}} d\left((1-t) \bar{v} \oplus t J_{\mu}^{f} \bar{v}, \bar{v}\right)^{p} \\
& \leq \frac{(1-t)}{p \mu^{p-1}} d(\bar{v}, \bar{v})^{p}+\frac{t}{p \mu^{p-1}} d\left(J_{\mu}^{f} \bar{v}, \bar{v}\right)^{p}-\frac{c t(1-t)}{2 p \mu^{p-1}} d\left(J_{\mu}^{f} \bar{v}, \bar{v}\right)^{p}
\end{aligned}
$$

which implies that

$$
\left(1+\frac{c t(1-t)}{2}-t\right) d\left(J_{\mu}^{f} \bar{v}, \bar{v}\right)^{p} \leq 0 .
$$

Since $t \neq 1$, we obtain that $\bar{v} \in F\left(J_{\mu}^{f}\right)$. Hence, $F\left(J_{\mu}^{f}\right)=\underset{y \in X}{\operatorname{argmin}} f(y)$.

We now recall important results which will be needed in the proofs of the main theorems of this subsection. 
Remark 3.9. Inequality (1.2) ensures that the function $x \mapsto d(., x)^{p}: X \rightarrow[0, \infty)$ is a convex and lower semicontinuous function.

Remark 3.10. [42],[19]. Let $X$ be a complete $p$-uniformly convex metric space. Then,

(i) every bounded sequence in $X$ has a unique asymptotic center,

(ii) every bounded sequence in $X$ has a $\Delta$-convergent subsequence.

Lemma 3.11. [50]. Let $\left\{a_{n}\right\}$ be a sequence of non-negative real numbers satisfying

$$
a_{n+1} \leq\left(1-\alpha_{n}\right) a_{n}+\alpha_{n} \delta_{n}+\gamma_{n}, n \geq 0,
$$

where $\left\{\alpha_{n}\right\},\left\{\delta_{n}\right\}$ and $\left\{\gamma_{n}\right\}$ satisfy the following conditions:

(i) $\left\{\alpha_{n}\right\} \subset[0,1], \Sigma_{n=0}^{\infty} \alpha_{n}=\infty$,

(ii) $\limsup _{n \rightarrow \infty} \delta_{n} \leq 0$,

(iii) $\gamma_{n} \geq 0(n \geq 0), \Sigma_{n=0}^{\infty} \gamma_{n}<\infty$.

Then $\lim _{n \rightarrow \infty} a_{n}=0$.

Lemma 3.12. For $1<p<\infty$, let $X$ be a complete p-uniformly convex metric space with parameter $c \geq 2$ and $f: X \rightarrow(-\infty,+\infty]$ a proper, convex and lower semicontinuous function. Let $\left\{\mu_{n}\right\}$ be a sequence of positive real numbers. Suppose $\lim _{n \rightarrow \infty} \mu_{n}=\infty$ and $A\left(\left\{J_{\mu_{n}}^{f} x_{n}\right\}\right)=\{\bar{v}\}$ for some bounded sequence $\left\{x_{n}\right\}$ of $X$. Then $\bar{v}$ is a minimizer of $f$, that is, $\bar{v} \in \underset{y \in X}{\operatorname{argmin}} f(y)$.

Proof. By Lemma 2.12, we obtain that

$$
\frac{c}{2}\left(\mu_{n}^{p-1}+1\right) d\left(J_{\mu_{n}}^{f} x_{n}, J^{f} \bar{v}\right)^{p}+d\left(J_{\mu_{n}}^{f} x_{n}, x_{n}\right)^{p}+\mu_{n}^{p-1} d\left(J^{f} \bar{v}, \bar{v}\right)^{p} \leq d\left(J^{f} \bar{v}, x_{n}\right)^{p}+\mu_{n}^{p-1} d\left(J_{\mu_{n}}^{f} x_{n}, \bar{v}\right)^{p},
$$

which implies

$$
\frac{c}{2} d\left(J_{\mu_{n}}^{f} x_{n}, J^{f} \bar{v}\right)^{p} \leq \frac{1}{\mu_{n}^{p-1}} d\left(J^{f} \bar{v}, x_{n}\right)^{p}+d\left(J_{\mu_{n}}^{f} x_{n}, \bar{v}\right)^{p} .
$$

By $\lim _{n \rightarrow \infty} \mu_{n}=\infty$ and $\left\{x_{n}\right\}$ is bounded, we obtain that

$$
\frac{c}{2} \limsup _{n \rightarrow \infty} d\left(J_{\mu_{n}}^{f} x_{n}, J^{f} \bar{v}\right)^{p} \leq \limsup _{n \rightarrow \infty} d\left(J_{\mu_{n}}^{f} x_{n}, \bar{v}\right)^{p} .
$$

Furthermore, since $A\left(\left\{J_{\mu_{n}}^{f} x_{n}\right\}\right)=\{\bar{v}\}$ and $c \geq 2$, we obtain that

$$
\limsup _{n \rightarrow \infty} d\left(J_{\mu_{n}}^{f} x_{n}, J^{f} \bar{v}\right) \leq \limsup _{n \rightarrow \infty} d\left(J_{\mu_{n}}^{f} x_{n}, \bar{v}\right)=\inf _{y \in X} \limsup _{n \rightarrow \infty} d\left(J_{\mu_{n}}^{f} x_{n}, y\right) .
$$

By (3.28), Remark 3.10 (i) and Lemma 3.8, we obtain that $\bar{v} \in F\left(J^{f}\right)=\underset{y \in X}{\operatorname{argmin}} f(y)$.

Theorem 3.13. For $1<p<\infty$, let $X$ be a complete p-uniformly convex metric space with parameter $c \geq 2$ and $f: X \rightarrow(-\infty,+\infty]$ a proper, convex and lower semicontinuous function. Let $\left\{x_{n}\right\}$ be the sequence defined by (3.26), where $\left\{\alpha_{n}\right\}$ is a sequence in $[0,1)$ and $\left\{\mu_{n}\right\}$ is a sequence in $(0, \infty)$ such that $\lim _{n \rightarrow \infty} \mu_{n}=\infty$. Then, the following hold:

(i) The sequence $\left\{J_{\mu_{n}}^{f} x_{n}\right\}$ is bounded if and only if $\underset{y \in X}{\operatorname{argmin}} f(y) \neq \emptyset$.

(ii) If $\lim _{n \rightarrow \infty} \alpha_{n}=0, \sum_{n=1}^{\infty} \alpha_{n}=\infty$ and $\operatorname{argmin}_{y \in X} f(y) \neq \emptyset$, then $\left\{x_{n}\right\}$ and $\left\{J_{\mu_{n}}^{f} x_{n}\right\}$ converge to an element of $\underset{y \in X}{\operatorname{argmin}} f(y)$.

Proof. (i) Suppose that $\left\{J_{\mu_{n}}^{f} x_{n}\right\}$ is bounded. Then by Remark 3.10 (i), there exists $\bar{v} \in X$ such that $A\left(\left\{J_{\mu_{n}}^{f} x_{n}\right\}\right)=$ $\{\bar{v}\}$. Thus, from (3.26) and Remark 3.9, we obtain that

$$
d\left(x_{n+1}, \bar{v}\right)^{p} \leq \alpha_{n} d(u, \bar{v})^{p}+\left(1-\alpha_{n}\right) d\left(J_{\mu_{n}}^{f} x_{n}, \bar{v}\right)^{p},
$$

which implies that $\left\{x_{n}\right\}$ is bounded. Also, since $\lim _{n \rightarrow \infty} \mu_{n}=\infty$ and $A\left(\left\{J_{\mu_{n}}^{f} x_{n}\right\}\right)=\{\bar{v}\}$, we obtain by Lemma 3.12 that $\bar{v}$ is a minimizer of $f$. Hence, $\underset{y \in X}{\operatorname{argmin}} f(y) \neq \emptyset$. 
Conversely, let $\underset{y \in X}{\operatorname{argmin}} f(y) \neq \emptyset$. Then, we may assume that $\bar{v}$ is a minimizer of $f$. Thus by (3.26), Remark 3.9 and Lemma 2.10, we obtain that

$$
\begin{aligned}
d\left(x_{n+1}, \bar{v}\right)^{p} & \leq \alpha_{n} d(u, \bar{v})^{p}+\left(1-\alpha_{n}\right) d\left(J_{\mu_{n}}^{f} x_{n}, \bar{v}\right)^{p} \\
& \leq \alpha_{n} d(u, \bar{v})^{p}+\left(1-\alpha_{n}\right) d\left(x_{n}, \bar{v}\right)^{p} \\
& \leq \max \left\{d(u, \bar{v})^{p}, d\left(x_{n}, \bar{v}\right)^{p}\right\}
\end{aligned}
$$

which implies by induction that

$$
d\left(x_{n}, \bar{v}\right)^{p} \leq \max \left\{d(u, \bar{v})^{p}, d\left(x_{1}, \bar{v}\right)^{p}\right\} \forall n \geq 1 .
$$

Therefore, $\left\{x_{n}\right\}$ is bounded. Consequently, $\left\{J_{\mu_{n}}^{f} x_{n}\right\}$ is also bounded.

(ii) Since $\underset{y \in X}{\operatorname{argmin}} f(y) \neq \emptyset$, we obtain from (3.29) that $\left\{x_{n}\right\}$ and $\left\{J_{\mu_{n}}^{f} x_{n}\right\}$ are bounded. Furthermore, we obtain by (1.2) and Lemma 2.10 that

$$
\begin{aligned}
d\left(x_{n+1}, \bar{v}\right)^{p} & \leq \alpha_{n} d(u, \bar{v})^{p}+\left(1-\alpha_{n}\right) d\left(J_{\mu_{n}}^{f} x_{n}, \bar{v}\right)^{p}-\frac{\alpha_{n}\left(1-\alpha_{n}\right) c}{2} d\left(u, J_{\mu_{n}}^{f} x_{n}\right)^{p} \\
& \leq \alpha_{n} d(u, \bar{v})^{p}+\left(1-\alpha_{n}\right) d\left(x_{n}, \bar{v}\right)^{p}-\alpha_{n}\left(1-\alpha_{n}\right) d\left(u, J_{\mu_{n}}^{f} x_{n}\right)^{p} \\
& =\left(1-\alpha_{n}\right) d\left(x_{n}, \bar{v}\right)^{p}+\alpha_{n} \delta_{n} \forall n \geq 1,
\end{aligned}
$$

where $\delta_{n}=d(u, \bar{v})^{p}+\left(\alpha_{n}-1\right) d\left(u, J_{\mu_{n}}^{f} x_{n}\right)^{p}$. Now, set $v_{n}=J_{\mu_{n}}^{f} x_{n} \forall n \geq 1$. Then, by the boundedness of $\left\{J_{\mu_{n}}^{f} x_{n}\right\}$, we obtain by Remark 3.10 (ii) that there exists a subsequence $\left\{v_{n_{k}}\right\}$ of $\left\{v_{n}\right\}$ that $\Delta$-converges to some $\hat{v} \in X$. Thus, by Remark 3.10 (i), we obtain that $A\left(\left\{v_{n_{k}}\right\}\right)=\{\hat{v}\}$. Moreover, $\lim _{k \rightarrow \infty} \mu_{n_{k}}=\infty$ and $\left\{x_{n_{k}}\right\}$ is bounded. Hence, by Lemma 3.12, we obtain that $\hat{v}$ is a minimizer of $f$.

Next, we show that $\left\{x_{n}\right\}$ converges to $\hat{v}$. Observe that

$$
d(u, \hat{v})^{p} \leq \liminf _{k \rightarrow \infty} d\left(u, v_{n_{k}}\right)^{p}=\lim _{k \rightarrow \infty} d\left(u, v_{n_{k}}\right)^{p}=\liminf _{n \rightarrow \infty} d\left(u, v_{n}\right)^{p} .
$$

Thus,

$$
\limsup _{n \rightarrow \infty} \delta_{n} \leq d(u, \hat{v})^{p}-\liminf _{n \rightarrow \infty} d\left(u, v_{n}\right)^{p} \leq 0 .
$$

Now, Lemma 3.11 applied to (3.30), gives that $\left\{x_{n}\right\}$ converges to $\hat{v}$.

In what follows, we intend to apply Theorem 3.13 to establish convergence of Halpern-type PPA (3.27) involving finite composition of resolvents of $f$.

Lemma 3.14. For $1<p<\infty$, let $X$ be a p-uniformly convex metric space with parameter $c \geq 2$ and $f: X \rightarrow$ $(-\infty,+\infty]$ a proper, convex and lower semicontinuous function. Then, for $\mu>0$, we have the following:

(i) $d\left(x^{*}, J_{\mu}^{f} x\right)^{p}+d\left(J_{\mu}^{f} x, x\right)^{p} \leq d\left(x^{*}, x\right)^{p}$ for all $x \in X$ and $x^{*} \in F\left(J_{\mu}^{f}\right)$ (where $F\left(J_{\mu}^{f}\right)$ denotes the set of fixed points of $\left.J_{\mu}^{f}\right)$;

(ii) $F\left(\prod_{j=1}^{m} J_{\mu}^{(j)}\right)=\cap_{j=1}^{m} F\left(J_{\mu}^{(j)}\right)$, where $\prod_{j=1}^{m} J_{\mu}^{(j)}=J_{\mu}^{f_{1}} \circ J_{\mu}^{f_{2}} \circ \cdots \circ J_{\mu}^{f_{m-1}} \circ J_{\mu}^{f_{m}}$.

Proof. (i) Let $x \in X$ and $x^{*} \in F\left(J_{\mu}^{f}\right)$. Then by setting $v=x^{*}$ in (2.3), we obtain that

$$
\frac{c}{2} d\left(J_{\mu}^{f} x, x^{*}\right)^{p} \leq p \mu^{p-1}\left(f\left(x^{*}\right)-f\left(J_{\mu}^{f} x\right)\right)+d\left(x^{*}, x\right)^{p}-d\left(J_{\mu}^{f} x, x\right)^{p} .
$$

Since $x^{*} \in F\left(J_{\mu}^{f}\right)$, therefore by Lemma 3.8 we obtain that $f\left(x^{*}\right) \leq f\left(J_{\mu}^{f} x\right)$. Hence, we obtain that

$$
d\left(x^{*}, J_{\mu}^{f} x\right)^{p}+d\left(J_{\mu}^{f} x, x\right)^{p} \leq d\left(x^{*}, x\right)^{p} .
$$

(ii) Clearly, $\cap_{j=1}^{m} F\left(J_{\mu}^{(j)}\right) \subseteq F\left(\prod_{j=1}^{m} J_{\mu}^{(j)}\right)$. Thus, we only have to show that $F\left(\prod_{j=1}^{m} J_{\mu}^{(j)}\right) \subseteq \cap_{j=1}^{m} F\left(J_{\mu}^{(j)}\right)$. For this, let $x \in F\left(\prod_{j=1}^{m} J_{\mu}^{(j)}\right)$ and $y \in \cap_{j=1}^{m} F\left(J_{\mu}^{(j)}\right)$, we obtain by Lemma 2.10 that

$$
\begin{aligned}
d(x, y)^{p} & =d\left(\prod_{j=1}^{m} J_{\mu}^{(j)} x, \prod_{j=1}^{m} J_{\mu}^{(j)} y\right)^{p} \\
& \leq d\left(\prod_{j=2}^{m} J_{\mu}^{(j)} x, y\right)^{p}
\end{aligned}
$$


Furthermore, we obtain by (i), Lemma 2.10 and (3.31) that

$$
\begin{aligned}
d\left(\prod_{j=2}^{m} J_{\mu}^{(j)} x, \prod_{j=1}^{m} J_{\mu}^{(j)} x\right)^{p} & \leq d\left(\prod_{j=2}^{m} J_{\mu}^{(j)} x, y\right)^{p}-d\left(\prod_{j=1}^{m} J_{\mu}^{(j)} x, y\right)^{p} \\
& \vdots \\
\leq & d(x, y)^{p}-d\left(\prod_{j=1}^{m} J_{\mu}^{(j)} x-y\right)^{p} \\
& =d\left(\prod_{j=1}^{m} J_{\mu}^{(j)} x, y\right)^{p}-d\left(\prod_{j=1}^{m} J_{\mu}^{(j)} x-y\right)^{p}
\end{aligned}
$$

which implies

$$
\prod_{j=1}^{m} J_{\mu}^{(j)} x=\prod_{j=2}^{m} J_{\mu}^{(j)} x
$$

Similarly, we obtain that

$$
\begin{aligned}
d\left(\prod_{j=3}^{m} J_{\mu}^{(j)} x, \prod_{j=2}^{m} J_{\mu}^{(j)} x\right)^{p} & \leq d\left(\prod_{j=3}^{m} J_{\mu}^{(j)} x, y\right)^{p}-d\left(\prod_{j=2}^{m} J_{\mu}^{(j)} x, y\right)^{p} \\
& \vdots \\
& \leq d(x, y)^{p}-d\left(\prod_{j=2}^{m} J_{\mu}^{(j)} x, y\right)^{p} \\
& \leq d\left(\prod_{j=1}^{m} J_{\mu}^{(j)} x, y\right)^{p}-d\left(\prod_{j=1}^{m} J_{\mu}^{(j)} x-y\right)^{p}
\end{aligned}
$$

which implies

$$
\prod_{j=2}^{m} J_{\mu}^{(j)} x=\prod_{j=3}^{m} J_{\mu}^{(j)} x
$$

Continuing in this manner, we can show that

$$
\prod_{j=3}^{m} J_{\mu}^{(j)} x=\prod_{j=4}^{m} J_{\mu}^{(j)} x=\cdots=\prod_{j=m-1}^{m} J_{\mu}^{(j)} x=J_{\mu}^{(m)} x=x .
$$

From (3.34), we have

$$
x=J_{\mu}^{f_{m}} x .
$$

From (3.34) and (3.35), we obtain

$$
x=\prod_{j=m-1}^{m} J_{\mu}^{(j)} x=J_{\mu}^{f_{m-1}} J_{\mu}^{f_{m}} x=J_{\mu}^{f_{m-1}} x .
$$

Continuing in this manner, we obtain from (3.32)-(3.36) that

$$
x=J_{\mu}^{f_{m-2}} x=\cdots=J_{\mu}^{f_{2}} x=J_{\mu}^{f_{1}} x .
$$

That is,

$$
J_{\mu}^{f_{1}} x=J_{\mu}^{f_{2}} x=\cdots=J_{\mu}^{f_{m-1}} x=J_{\mu}^{f_{m}} x=x .
$$

Hence, we obtain the desired conclusion. 
Theorem 3.15. For $1<p<\infty$, let $X$ be a complete $p$-uniformly convex metric space with parameter $c \geq 2$ and $f_{j}: X \rightarrow(-\infty,+\infty]$ be proper, convex and lower semicontinuous functions. Let $\left\{x_{n}\right\}$ be a sequence generated by (3.27), where $\left\{\alpha_{n}\right\}$ is a sequence in $[0,1)$ and $\left\{\mu_{n}\right\}$ is a sequence in $(0, \infty)$ such that $\lim _{n \rightarrow \infty} \mu_{n}=\infty$. If $\lim _{n \rightarrow \infty} \alpha_{n}=0, \sum_{n=1}^{\infty} \alpha_{n}=\infty$ and $\Gamma:=\cap_{j=1}^{m} \operatorname{argmin}_{y \in X} f_{j}(y) \neq \emptyset$, then the sequence $\left\{x_{n}\right\}$ converges to an element of $\Gamma$.

Proof. By Theorem 3.13 (ii) and Lemma 3.8, we obtain that $\left\{x_{n}\right\}$ converges to an element of $F\left(\prod_{j=1}^{m} J_{\mu}^{f_{j}}\right)$. Therefore, we conclude by Lemma 3.14 (ii) and Lemma 3.8 that $\left\{x_{n}\right\}$ converges to an element of $\Gamma$.

Corollary 3.16. Let $X$ be a complete 2-uniformly convex metric space (in particular, complete CAT(0) space) and $f_{j}: X \rightarrow(-\infty,+\infty]$ be proper, convex and lower semicontinuous functions. Let $\left\{x_{n}\right\}$ be a sequence generated by (3.27), where $\left\{\alpha_{n}\right\}$ is a sequence in $[0,1)$ and $\left\{\mu_{n}\right\}$ is a sequence in $(0, \infty)$ such that $\lim _{n \rightarrow \infty} \mu_{n}=\infty$. If $\lim _{n \rightarrow \infty} \alpha_{n}=0, \sum_{n=1}^{\infty} \alpha_{n}=\infty$ and $\Gamma:=\cap_{j=1}^{m} \underset{y \in X}{\operatorname{argmin}} f_{j}(y) \neq \emptyset$, then the sequence $\left\{x_{n}\right\}$ converge to an element of $\Gamma$.

Proof. Take $p=2=c$ in Theorem 3.15 .

\section{NumericAl EXAMPLES}

Let $X=\mathbb{R}^{4}$ be endowed with the Euclidean norm. For $x=\left(x_{1}, x_{2}, x_{3}, x_{4}\right) \in X$, define $f, g: X \rightarrow(-\infty, \infty]$ by

$$
f(x)=\frac{1}{2}\|A(x)-a\|_{2}^{2}, g(x)=\frac{1}{2}\|B(x)-b\|_{2}^{2},
$$

where

$$
\begin{aligned}
A & =\left[\begin{array}{cccc}
3 & 1 & -2 & 2 \\
1 & 3 & 4 & 5 \\
2 & 3 & 1 & 4 \\
5 & 2 & 3 & 1
\end{array}\right] \text { and } a=\left[\begin{array}{c}
-1 \\
3 \\
5 \\
4
\end{array}\right], \\
B & =\left[\begin{array}{cccc}
2 & 3 & 1 & -1 \\
4 & -4 & 3 & 2 \\
-1 & 3 & 2 & -4 \\
5 & 7 & 4 & 3
\end{array}\right] \text { and } b=\left[\begin{array}{l}
3 \\
0 \\
1 \\
2
\end{array}\right]
\end{aligned}
$$

Then, $f$ and $g$ are proper convex and lower semicontinuous functions (see [17, 32]). Thus, by [32], we know that

$$
J_{1}^{f}(x)=\operatorname{prox}^{f}(x)=\left(I+A^{t} A\right)^{-1}\left(x+A^{t} a\right) \text { and } J_{1}^{g}(x)=\operatorname{prox}^{g}(x)=\left(I+B^{t} B\right)^{-1}\left(x+B^{t} b\right) .
$$

Hence, the BBA (3.1) (studied in this paper) becomes

$$
\left\{\begin{array}{l}
y_{n}=\left(I+B^{t} B\right)^{-1}\left(x_{n}+B^{t} b\right), \\
x_{n+1}=\left(I+A^{t} A\right)^{-1}\left(y_{n}+A^{t} a\right), n \geq 1,
\end{array}\right.
$$

the classical PPA (1.4) (studied by Choi and Ji [14]) becomes

$$
x_{n+1}=\left(I+A^{t} A\right)^{-1}\left(x_{n}+A^{t} a\right), n \geq 1,
$$

the Halpern-type PPA (3.27) (studied in this paper) becomes

$$
x_{n+1}=\alpha_{n} u+\left(1-\alpha_{n}\right) J_{1}^{f}\left(J_{1}^{g} x_{n}\right), n \geq 1,
$$

where $\alpha_{n}=\frac{1}{10(n+1)}, \forall n \geq 1$,

and the hybrid PPA in [49, Algorithm 3.1] becomes

$$
\left\{\begin{array}{l}
z_{n}=\left(I+A^{t} A\right)^{-1}\left(x_{n}+A^{t} a\right), \\
y_{n}=\left(1-\alpha_{n}\right) z_{n}, \\
x_{n+1}=\left(1-\beta_{n}\right) z_{n}+\beta_{n} y_{n}, n \geq 1,
\end{array}\right.
$$

where $\alpha_{n}=\frac{1}{10(n+1)}$ and $\beta_{n}=\frac{3 n-1}{50 n}$ for all $n \geq 1$.

Case 1: Take $x_{1}=(1,0.5,-1,1)^{t}$. 
Case 2: Take $x_{1}=(-1,2,-1,3)^{t}$.

Case 3: Take $x_{1}=(6,7,9,11)^{t}$.

Case 4: Take $x_{1}=(-6,-2,-0.2,-0.5)^{t}$.

Using different choices of the initial vector $x_{1}$ (that is, Case 1-Case 4), we compared Algorithms (4.1)-(4.4) as shown in the graphs and table below. Notice that we only considered the table for Case $\mathbf{1}$ since the tables for other cases are similar to it. The graphs and table show that our algorithms (Algorithms (4.1) and (4.3)) converges faster than Algorithms (4.2) and (4.4) studied by Choi and Ji [14], and Ugwunnadi et. al. [48] respectively. 
Table 1. Numerical results for BBA (4.1)

\begin{tabular}{|c|c|c|}
\hline No. of iterations & $x_{n}=\left(x_{n 1}, x_{n 2}, x_{n 3}, x_{n}\right)^{t}$ & Errors $=|| x_{n}-x_{n-1}||_{2}$ \\
\hline 1 & $(1.0000,0.5000,-1.0000,1.0000)$ & \\
\hline 2 & $(0.71350 .2351,-0.2993,-0.5831)$ & 1.7746 \\
\hline 3 & $(0.7233,0.2384,-0.3074,-0.5963)$ & 0.0186 \\
\hline 4 & $(0.7237,0.2385,-0.3077,-0.5966)$ & 0.0006 \\
\hline 5 & $(0.7237,0.23852,-0.3077,-0.5967)$ & 0.0000 \\
\hline 6 & $(0.7237,0.2385,-0.3077,-0.5967)$ & 0.0000 \\
\hline 7 & $(0.7237,0.2385,-0.3077,-0.5967)$ & 0.0000 \\
\hline
\end{tabular}

Table 2. Numerical results for the classical PPA (4.2) of Choi and Ji

\begin{tabular}{|c|c|c|}
\hline No. of iterations & $x_{n}=\left(x_{n 1}, x_{n 2}, x_{n 3}, x_{n} 4\right)^{t}$ & Errors $=|| x_{n}-\left.x_{n-1}\right|_{2}$ \\
\hline 1 & $(1.0000,0.5000,-1.0000,1.0000)$ & \\
\hline 2 & $(0.0853,1.3349,0.3046,-0.2646)$ & 2.1988 \\
\hline 3 & $(-0.1074,2.1758,0.2707,-0.7311)$ & 0.9814 \\
\hline 4 & $(-0.2410,2.8344,0.1748,-1.0603)$ & 0.7544 \\
\hline 5 & $(-0.3439,3.3444,0.0962,-1.3126)$ & 0.5836 \\
\hline 6 & $(-0.4236,3.7390,0.0350,-1.5077)$ & 0.4514 \\
\hline. & & \\
\hline. & & \\
\hline. & $(-0.6956,5.0868,-0.1739,-2.1738)$ & 0.0001 \\
\hline 41 & $(-0.6956,5.0868,-0.1739,-2.1738)$ & 0.0000 \\
\hline 42 & $(-0.6956,5.0868,-0.1739,-2.1738)$ & 0.0000 \\
\hline 43 & &
\end{tabular}


Table 3. Numerical results for Halpern-type PPA (4.3)

\begin{tabular}{|c|c|c|}
\hline No. of iterations & $\mathrm{x}_{\mathrm{n}}=\left(\mathrm{x}_{\mathrm{n} 1}, \mathrm{x}_{\mathrm{n} 2}, \mathrm{x}_{\mathrm{n} 3}, \mathrm{x}_{\mathrm{n} 4}\right)^{\mathrm{t}}$ & Errors $=|| \mathrm{x}_{\mathrm{n}}-\mathrm{x}_{\mathrm{n}-1} \|_{2}$ \\
\hline 1 & $(1.0000,0.5000,-1.0000,1.0000)$ & \\
\hline 2 & $(0.0513,1.5315,0.3144,-0.4422)$ & 2.4024 \\
\hline 3 & $(0.0444,1.5491,0.3271,-0.4470)$ & 0.0233 \\
\hline 4 & $(0.0439,1.5615,0.3292,-0.4516)$ & 0.0133 \\
\hline 5 & $(0.0436,1.5691,0.3304,-0.4545)$ & 0.0083 \\
\hline 6 & $(0.0433,1.5742,0.3311,-0.4564)$ & 0.0055 \\
\hline. & & \\
\hline. & & 0.0000 \\
\hline. & $(0.0425,1.5960,0.3344,-0.4647)$ & 0.0000 \\
\hline 9 & $(0.0425,1.5960,0.3344,-0.4647)$ & 0.0000 \\
\hline 10 & $(0.0425,1.5960,0.3344,-0.4647)$ & \\
\hline 11 & & \\
\hline
\end{tabular}

Table 4. Numerical results for Hybrid PPA (4.4)

\begin{tabular}{|c|c|c|}
\hline No. of iterations & $\mathrm{x}_{\mathrm{n}}=\left(\mathrm{x}_{\mathrm{n} 1}, \mathrm{x}_{\mathrm{n} 2}, \mathrm{x}_{\mathrm{n} 3}, \mathrm{x}_{\mathrm{n} 4}\right)^{\mathrm{t}}$ & Errors $=|| \mathrm{x}_{\mathrm{n}}-\mathrm{x}_{\mathrm{n}-1} \|_{2}$ \\
\hline 1 & $(1.0000,0.5000,-1.0000,1.0000)$ & \\
\hline 2 & $(0.0853,1.3349,0.3046,-0.2646)$ & 2.1988 \\
\hline 3 & $(-0.1002,2.0308,0.2526,-0.6824)$ & 0.8343 \\
\hline 4 & $(-0.2054,2.5399,0.1752,-0.9386)$ & 0.5847 \\
\hline 5 & $(-0.2809,2.9218,0.1191,-1.1259)$ & 0.4357 \\
\hline 6 & $(-0.3386,3.2175,0.0771,-1.2700)$ & 0.3366 \\
\hline. & & \\
\hline. & & \\
\hline 221 & $(-0.6866,5.0385,-0.1678,-2.1507)$ & 0.0003 \\
\hline 222 & $(-0.6867,5.0387,-0.1678,-2.1508)$ & 0.0002 \\
\hline 223 & $(-0.6867,5.0389,-0.1679,-2.1509)$ & 0.0002 \\
\hline
\end{tabular}



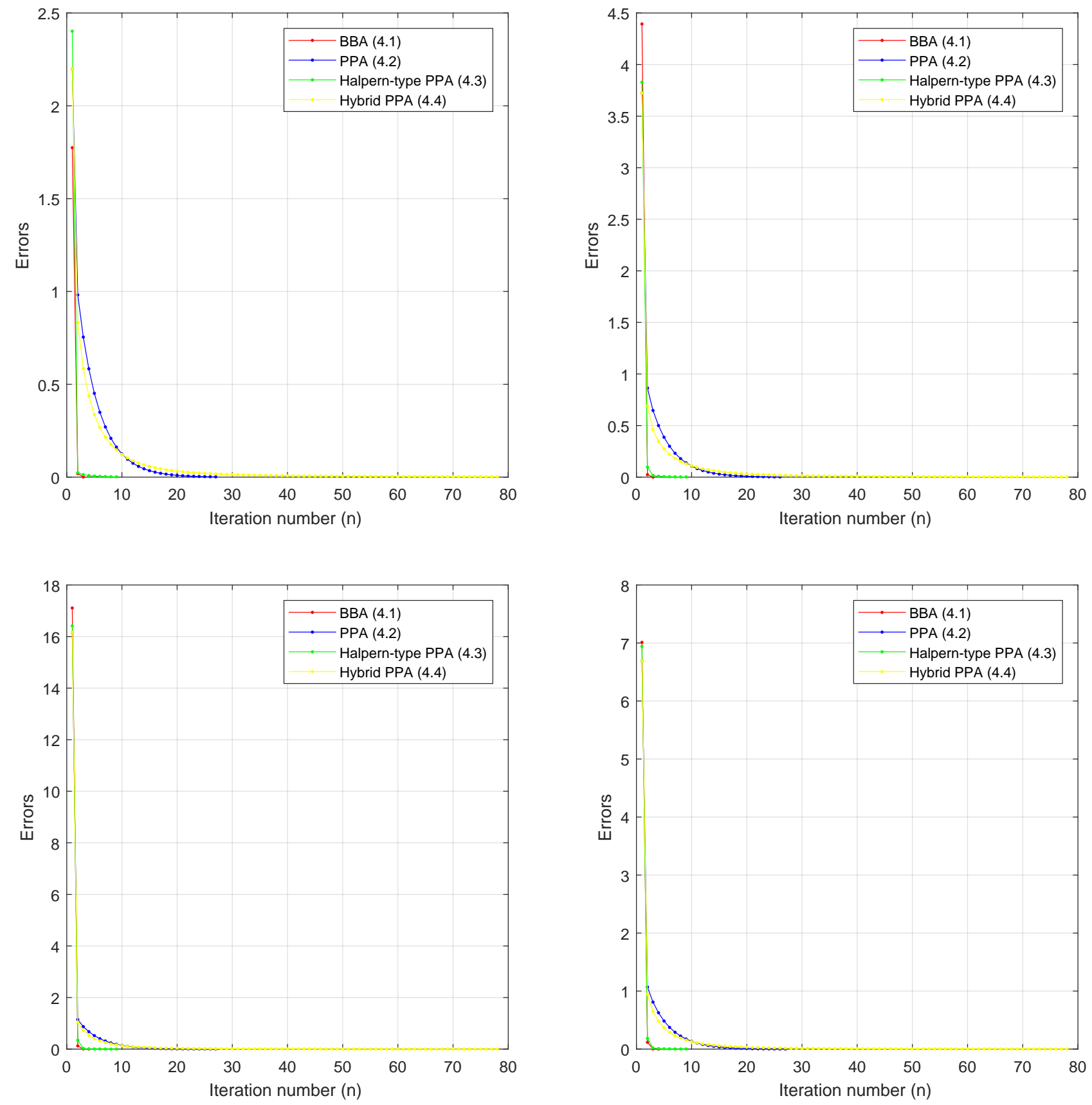

Figure 1. Errors vs Iteration numbers(n): Case 1 (top left); Case 2 (top right); Case 3 (bottom left); Case 4 (bottom right).

\section{Declaration}

The authors declare that they have no competing interests.

\section{REFERENCES}

[1] H. Attouch, J. Bolte, P. Redont A. Soubeyran, Proximal alternating minimization and projection methods for nonconvex problems: An approach based on the Kurdyka-Lojasiewicz inequality, Math. Oper. Res., 35, (2010) 438-457.

[2] H. Attouch, J. Bolte, P. Redont A. Soubeyran, Alternating proximal point algorithms for weakly coupled convex minimization problems. Applications to dynamical gam es and PDE's, J. Convex Anal., 15 (3) (2008), 485-506.

[3] H. Attouch, J. Bolte, P. Redont A. Soubeyran, A new class of alternating proximal minimization algorithms with costs to move, SIAM J. Optim. 18 (3) (2007), 1061-1081. 
[4] H. A. Abass, C. Izuchukwu, F. U. Ogbuisi, O.T. Mewomo, An iterative method for solution of finite families of split minimization problems and fixed point problems, Novi Sad J. Math, (2018). Doi:10.30755/NSJOM.07925.

[5] M. Bačák, Computing medians and means in Hadamard spaces, SIAM J. Optim., 24 (2014), 1542-1566.

[6] M. Bačák, The proximal point algorithm in metric spaces, Israel J. Math., 194 (2013), 689-701.

[7] K. Ball, E. A. Carlen, E. H. Lieb, Sharp uniform convexity and smoothness inequalities for trace norms, Invent. Math., 115 (1994), 463-482.

[8] S. Banert, Backward-Backward splitting in Hadamard spaces, J. Math. Anal. Appl., 414 (2014), 656-665.

[9] H. Bauschke, J. Burke, F. Deutsch, H. Hundal, J. Vanderwerff, A new proximal point iteration that converges weakly but not in norm, Proc. Amer. Math. Soc., 133 (2005), 1829-1835.

[10] H. Bauschke, E. Matoušková, S. Reich, Projection and proximal point methods: convergence results and counterexamples, Nonlinear Anal., 56 (2004), 715-738.

[11] I. D. Berg and I. G. Nikolaev, Quasilinearization and curvature of Alexandrov spaces, Geom. Dedicata, 133 (2008), $195-218$.

[12] M. R. Bridson and A. Haefliger, Metric spaces of non-positive Curvature, Fundamental Principle of Mathematical Sciences, Springer, Berlin, Germany, 319 (1999).

[13] A. Cabot and P. Frankel, Alternating proximal algorithms with asymptotically vanishing coupling. Application to domain decomposition for PDE's, Optimization, 61 (3) (2012), 307-325.

[14] B. J. Choi and U. C. Ji, The proximal point algorithm in uniformly convex metric spaces, Commun. Korean Math. Soc., 31 (2016), 845-855.

[15] J.X. Cruz Neto, B.P. Lima, P.A. Soares Júnior, A splitting minimization method on geodesic spaces, (2013). Optimizationonline.org/DB_FILE/2013/04/3822.pdf

[16] J.X. Cruz Neto, P.R. Oliveira, P.A. Soares Júnior, A. Soubeyran, Learning how to play Nash, potential games and alternating minimization method for structured nonconvex problems on Riemannian manifolds, J. Convex Anal. 20 (2013).

[17] P.L. Combettes and J.C. Pesquet, Proximal splitting methods in signal processing, arXiv:0912.3522v4 [math. OC] (2010)

[18] S. Dhompongsa and B. Panyanak, On $\Delta$-convergence theorems in CAT(0) spaces, Comput. Math. Appl., 56 (2008), $2572-2579$.

[19] R. Espínola, A. Fernández-León, B. Piatek, Fixed points of single-valued and set-valued mappings in uniformly convex metric spaces with no metric convexity, Fixed Point Theory Appl., 2010 (2010).

[20] O. P. Ferreira and P. R. Oliveira, Proximal point algorithm on Riemannian manifolds, Optimization, 51 (2) (2002), 257-270.

[21] O. Güler, On the convergence of the proximal point algorithm for convex minimization, SIAM J. Control Optim., 29 (1991), 403-419

[22] L. O. Jolaoso, F. U. Ogbuisi, O. T. Mewomo, An iterative method for solving minimization, variational inequality and fixed point problems in reflexive Banach spaces, Adv. Pure Appl. Math., 9(3)(2017), 167 - 184.

[23] L.O. Jolaoso, K.O. Oyewole, C.C. Okeke, O.T. Mewomo, A unified algorithm for solving split generalized mixed equilibrium problem and fixed point of nonspreading mapping in Hilbert space, Demonstr. Math., 51, (2018), 211-232.

[24] S. Kamimura and W. Takahashi, Approximating solutions of maximal monotone operators in Hilbert spaces, J. Approx. Theory, 106 (2000), 226-240.

[25] M. Kell, Uniformly convex metric spaces, Anal. Geom. Metr. Spaces, DOI: 10.2478/agms-2014-0015 (2014).

[26] F. Kohsaka and W. Takahashi, Fixed point theorems for a class of nonlinear mappings related to maximal monotone operators in Banach spaces, Arch. Math., 91 (2008), 166-177.

[27] Y. Kimura and F. Kohsaka, Two modified proximal point algorithm for convex functions in Hadamard spaces, Linear and Nonlinear Anal., 2 (1) (2016), 69-86.

[28] K. Kuwae, Resolvent flows for convex functionals and p-Harmonic maps, Anal. Geom. Metr. Spaces, 3 (2015), 46-72.

[29] K. Kuwae, Jensen's inequality on convex spaces, Calc. Var. Partial Differential Equations 49 (2014), no. 3-4, $1359-1378$.

[30] C. Li, G. López, V. Martín-Márquez, Monotone vector fields and the proximal point algorithm on Hadamard manifolds, J. Lond. Math. Soc., 79 (2009), 663-683.

[31] T. C. Lim, Remarks on some fixed point theorems, Proc. Amer. Math. Soc., 60 (1976), 179-182.

[32] B. Martinet, Régularisation d'Inéquations Variationnelles par Approximations Successives, Rev.Franćaise dInform. et de Rech. Opérationnelle 3 (1970), 154-158.

[33] O.T. Mewomo and F.U. Ogbuisi, Convergence analysis of an iterative method for solving multiple-set split feasibility problems in certain Banach spaces, Quest. Math., 41(1), (2018), 129-148.

[34] A. Naor and L. Silberman, Poincaré inequalities, embeddings, and wild groups, Compos. Math., 147 (2011), $1546-1572$.

[35] F.U. Ogbuisi and O.T. Mewomo, Iterative solution of split variational inclusion problem in a real Banach space, Afr. Mat., 28(1-2), (2017), 295-309.

[36] S. Ohta, Markov type of Alexandrov spaces of nonnegative curvature, arXiv 0707.0102v3 [Math].MG], (2010).

[37] S. Ohta, Uniform convexity and smoothness, and their applications in Finsler geometry, Mathematische Annalen, 343 (2009), 669-699.

[38] S. Ohta, Convexities of metric spaces, Geom. Dedicata, 125 (2007), 225-250.

[39] S. Ohta, Regularity of harmonic functions in Cheeger-type Sobolev spaces, Ann. Global Anal. Geom., 26 (2004), $397-410$.

[40] E. P. Quiroz and P. Oliveira, Proximal point methods for quasiconvex and convex functions with Bregman distances on Hadamard manifolds, J. Convex Anal., 16 (2009), 49-69.

[41] R. T. Rockafellar, Monotone operators and the proximal point algorithm, SIAM J. Control Optim., 14 (1976), 877-898.

[42] D. A. Ruiz, G. L. Acedo, A. Nicolae, The asymptotic behavior of the composition of firmly nonexpansive mappings, arXiv:1411.6779v1 [math.FA] 25 Nov. 2014.

[43] Y. Shehu and O.T. Mewomo, Further investigation into split common fixed point problem for demicontractive operators, Acta Math. Sin. (Engl. Ser.), 32 (11) (2016), 1357-1376.

[44] Y. Shehu, O. T. Mewomo, F. U. Ogbuisi, Further investigation into approximation of a common solution of fixed point problems and split feasibility problems, Acta. Math. Sci. Ser. B, Engl. Ed., 36 (3) (2016), 913-930.

[45] Z. Shen, Lectures on Finsler geometry, World Scientific Publishing Co., Singapore, (2001). 
[46] K. T. Sturm, Probability measures on metric spaces of nonpositive curvature, Heat kernels and analysis on manifolds, graphs, and metric spaces (Paris, 2002), 357-390, Contemp. Math., 338, Amer. Math. Soc., Providence, RI, (2003).

[47] W. Takahashi, A convexity in metric space and nonexpansive mappings, I, Kōodai Math. Sem. Rep., 22 (1970), $142-149$.

[48] G. C. Ugwunnadi C. Izuchukwu, O. T. Mewomo, Strong convergence theorem for monotone inclusion problem in CAT(0) spaces Afr. Mat., (2018), https://doi.org/10.1007/s13370-018-0633-x.

[49] G. C. Ugwunnadi, A. R. Khan and M. Abbas, A hybrid proximal point algorithm for finding minimizers and fixed points in CAT(0) spaces, J. Fixed Point Theory Appl., (2018), DOI 10.1007/s11784-018-0555-0.

[50] H. K. Xu, Iterative algorithms for nonlinear operators, J. London. Math. Soc., 2(2002), 240-256.

1,3 School of Mathematics, Statistics and Computer Science, University of KwaZulu-Natal, Durban, South Africa.

2 Department of Mathematics, University of Eswatini, Private Bag Kwaluseni, Eswatini.

4 Department of Mathematics and Statistics, King Fahd University of Petroleum and Minerals, Dhahran, Saudi Arabia.

5 Department of Mathematics, Government College University, Lahore, Pakistan ., Department of Mathematics and Applied Mathematics, University of Pretoria, Pretoria, South Africa.

E-mail address: ${ }^{1}$ izuchukwu_c@yahoo.com, izuchukwuc@ukzn.ac.za

E-mail address: ${ }^{2}$ ugwunnadi4u@yahoo.com, gcugwunnadi@uniswa.sz

E-mail address: ${ }^{3}$ mewomoo@ukzn.ac.za

E-mail address: ${ }^{4}$ arahim@kfump.edu.sa

E-mail address: ${ }^{5}$ abbas.mujahid@gmail.com 